\title{
SIGNATURES OF DYNAMICAL STAR FORMATION IN THE OPHIUCHUS ASSOCIATION OF PRE-MAIN-SEQUENCE STARS
}

\author{
VALERI V. MaKarov \\ Michelson Science Center, California Technology Institute, 770 South Wilson Avenue, MS 100-22, Pasadena, CA 91125; vvm@caltech.edu \\ Received 2007 May 29; accepted 2007 August 16
}

\begin{abstract}
A sample of 58 probable members of the association of pre-main-sequence stars around the filamentary $\rho$ Ophiuchi cloud is investigated. Using astrometric proper motions from the UCAC-2 catalog and the convergent point method, the total heliocentric systemic velocity is estimated at $19 \mathrm{~km} \mathrm{~s}^{-1}$, and the mean distance at $145 \mathrm{pc}$. A small, statistically insignificant difference between the geometric convergent point and the actual direction of velocity defined by the observed radial velocity implies a small, if any, rate of expansion of the association. The Ophiuchus association appears to have a considerable depth, with half of the members lying within $15 \mathrm{pc}$ of the center. When the moving cluster distances are taken into account, the near-infrared $M_{K s}$ versus $\left(J-K_{s}\right) \mathrm{H}$-R diagram reveals an apparent large spread of ages between $14 \mathrm{Myr}$ and younger than $1 \mathrm{Myr}$. Most of the youngest stars are located along a slightly curved strip just south of the densest cloud, extending in the east-west direction roughly aligned with the central streamer of warm dust. The intersection of this strip with a thin segment of shocked dust visible in the IRAS $12 \mu \mathrm{m}$ map at $(\alpha, \delta)=\left(16^{\mathrm{h}} 31^{\mathrm{m}},-24.5^{\circ}\right)$ is marked with a small cluster of probably very young stars around ROXs $43 \mathrm{~A}$. The large extent and depth of the association, the moderate rate of expansion, the spread in ages of about $14 \mathrm{Myr}$, and the alignment of very young stars with the dusty streamer point at a dynamical mode of star formation in this region, scattered far and wide around the main core.
\end{abstract}

Subject headings: open clusters and associations: individual (Ophiuchus) — stars: formation — stars: kinematics — stars: pre-main-sequence

\section{INTRODUCTION}

The nearby star-forming region in Ophiuchus, traditionally associated with the embedded and highly reddened star $\rho \mathrm{Oph}$, is characterized by a very dense central cloud L1688 (Wilking \& Lada 1983), and a complex of extended, filament-like nebulae (streamers) bearing signs of a highly dynamical environment. The central cloud harbors a cluster of young stellar objects with a median age of 0.3 Myr (Greene \& Meyer 1995; Luhman \& Rieke 1999), which are dimmed by the ambient dust to such extent that they can be seen only in the infrared, millimeter or radio wavelengths. Until recently, these forming stars drew most of the attention, while the somewhat older stars outside the main cloud remained largely unobserved. The advent of global X-ray surveys, especially that of ROSAT, spurred interest in the extensive associations of classical and weak-lined T Tauri stars (CTTSs and WTTSs, respectively) surrounding the compact regions of ongoing star formation. The young X-ray associations, e.g., in Orion and Corona Australis, turned out to be large in angular extent, occupying tens or even hundreds of square degrees. A considerable number of widely dispersed WTTS members have been identified, which can hardly be as young as the embedded stars and young stellar objects. Could it be that the star-forming cores we observe today have been stable and fertile for 10-20 Myr, continuously generating and ejecting new stars? This scenario would be consistent with the standard model of star formation predicated on the existence of stable, long-lived cores of gradually decreasing internal magnetic pressure from the ambipolar diffusion (Mouschovias et al. 2006). In the previous paper (Makarov 2007a), I investigated the greater association of pre-main-sequence stars in Lupus, identified from X-ray and spectroscopic observations by other authors. Using the available astrometric data and the classical convergent point method, it was shown that the members span the range 1-28 Myr in age, and that the rate of expansion is too low for the association as a whole to fill in the current volume from a significantly more compact configuration. Therefore, even the oldest members were formed close to their present-day locations, sometimes tens of parsecs away from the active cores. The first generations of stars in Lupus were likely born in extended formations scattered in space. And yet, the greater association is surprisingly coherent kinematically, as advertised by the similarity of proper motions. At first glance, this makes a confusing picture: a large and generally sparse cloud with little evidence of internal turbulence lives through multiple short bursts of star formation at various locations, spanning almost $30 \mathrm{Myr}$ in time.

The main objective of this paper is to find out if the greater Ophiuchus association is also the result of this large-scale, multipleepoch star formation mechanism. This paper differs from previous investigations in the literature in that a much broader search for possible members of the Ophiuchus association is attempted, and that only stars with accurate astrometric proper motions are considered in order to determine their space motion and estimate the distances. The set of stars considered in this paper as probable members of the Ophiuchus association occupy roughly an area of $25 \mathrm{deg}^{2}$, and slightly overlap with the adjoining Upper Scorpius $\mathrm{OB}$ association. The photometric and imaging data from the 2MASS near infrared survey is also extensively used to derive the spread in ages. Understanding of the present and past conditions in Ophiuchus is important, because it (rather than the Pleiades, for example) is the paragon of the local star formation from our current viewpoint in the Galaxy. Quite a few nearby star-forming regions and young clusters display similar properties to Ophiuchus, such as IC 2602, IC 2391, R CrA, and Lupus. They are located inside, or physically and kinematically connected to the greater Sco-Cen belt, have extended halos of comoving stars, and exhibit a finite range of stellar ages.

\section{DATA AND METHOD}

Using the Simbad and Vizier World Wide Web facilities at CDS, a search for probable members of the Ophiuchus association was 
undertaken within approximately $3^{\circ}$ of the star $\rho$ Oph. Candidate members were cross-identified with the entries in the second release of the UCAC catalog (Zacharias et al. 2004). Only stars with proper motions in this catalog are considered in this paper, which effectively sets the faint magnitude limit of the sample and the angular resolution in double stars. The selected 58 astrometric members are listed in Table 1, along with their equatorial International Celestial Reference System (ICRS) coordinates, proper motions from the UCAC2 catalog, $J$ and $K_{s}$ magnitudes from the 2MASS near-infrared survey, and other quantities derived in this paper. The sample is not complete in any sense, being mostly derived from the previous spectroscopic and photometric studies in the literature, which usually covered much smaller areas on the sky. The bulk of standard proper motion errors are in the range 1-12 mas $\mathrm{yr}^{-1}$ per coordinate direction. The selected probable members have similar proper motions with an average of roughly $\left(\mu_{\alpha *}, \mu_{\delta}\right)=(-11.4,-24.5)$ mas $\mathrm{yr}^{-1}$. Like the Lupus pre-mainsequence association (Makarov 2007a), the Ophiuchus members are involved in a common stream-like motion, most of which is just the reflex standard solar motion. This fact allows me to use the powerful convergent point method, traditionally applied to open clusters. In the absence of isotropic expansion or contraction, the geometrically determined convergent point is the true direction of the systemic velocity of the cluster. The classical convergent point relations can yield, for example, distances from spectroscopically measured radial velocities, leading in many cases to better defined H-R diagrams than assuming the same mean distance to all members (e.g., de Bruijne 1999; de Bruijne et al. 2001). This improvement becomes crucial for young associations in star-forming regions, which have large dimensions in depth, as well as in the sky projection.

The technique of convergent point determination from proper motion vectors employed in this paper is described in detail in a few previous papers (Makarov \& Robichon 2001; Makarov 2006). It is based entirely on the directly observed astrometric data (positions and proper motions). The quadratic sum of weighted angular deviations of proper vector directions from the estimated convergent point, $\sum_{i}\left(\Delta_{i} / \sigma_{\Delta_{i}}\right)^{2}$, is the estimator in this Amoebabased nonlinear optimization. Since only the directions of proper motion vectors are utilized at this stage, individual distances can be estimated from the proper motion magnitudes independently in a statistically consistent way. If the cluster undergoes expansion or contraction, the geometric convergent point shifts with respect to the true direction of motion away from the cluster or toward it, respectively. This displacement can be detected only if the radial velocity of the cluster as a whole is known (usually, from spectroscopy). Unfortunately, Ophiuchus and other associations infringing or lying inside the Sco-Cen belt, have convergent points at $\lambda \approx 90^{\circ}$ on the sky (in other terms, have small radial velocities), where $\lambda$ is the angular separation between the geometrical center of the association and the convergent point. This makes the convergent point error along the overall proper motion direction large, and the detection of the displacement not as definitive as it could have been otherwise. An overall rotation of the cluster can also be detected from a sidewise displacement of the convergent point (Makarov 2006), but this requires a large number of members and a large angular extent on the sky.

\section{CONVERGENT POINT}

The sky position and the proper motions of the selected stars are depicted in Figure 1. The first impression from the proper motion field is that the association includes two subgroups of slightly different proper motion directions, which reflect mostly in the $\alpha \cos \delta$ component. Unfortunately, the modest astrometric precision for the optically dim stars prevents a statistically sound proof of this bifurcation. This first hint at kinematical segregation in a very young association should be verified in the future when more accurate astrometry becomes available. The inset in Figure 1 shows the estimated position of the convergent point and the $1 \sigma$ error ellipse computed from the covariance matrix. The ICRS position of the convergent point is $(\alpha, \delta)=\left(100^{\circ},-42^{\circ}\right) \pm$ $\left(25^{\circ}, 29^{\circ}\right)$. The error ellipse is strongly elongated along the great circle connecting the geometric center of the association with the convergent point, because of the relatively small angular size of our sample and the larger than expected dispersion of residuals. The largest error comes in the estimation of the angular distance between the center and the convergent point, i.e., $\lambda=107^{\circ} \pm$ $34^{\circ}$. The error in the transverse dimension is only $1^{\circ}$.

Eighteen stars in Table 1 have parallax measurements in the Hipparcos catalog (ESA 1997). The median relative precision of these parallaxes is about $15 \%$. For the rest of the membership, parallaxes are not available, and the moving cluster method provides the only estimates of distances. This is achieved in two steps; first, the 18 trigonometric parallaxes are used to determine the centroid heliocentric velocity of the association. It turns out to be $19 \mathrm{~km} \mathrm{~s}^{-1}$. It is then assumed that all the members have the same velocity in space, and the convergent point formula is reversed for each star to produce a distance. These "kinematic distances" are specified in Table 1. Two kinds of stochastics contribute to the uncertainties of these distances: (1) the astrometric measurement errors, and (2) the intrinsic dispersion of velocities, discussed below. The relative precision of the kinematical distances is expected to be $10 \%$ or better for $\sim 60 \%$ of the selected members, but for several dimmer stars, it deteriorates to nearly $50 \%$. This calls for caution when discussing the results for stars with proper motion errors $\gtrsim 5$ mas $\mathrm{yr}^{-1}$.

The mean radial velocity of the Ophiuchus stars is $+2.2 \mathrm{~km} \mathrm{~s}^{-1}$ (de Zeeuw et al. 1999). Since the mean heliocentric velocity is $19 \mathrm{~km} \mathrm{~s}^{-1}$, the estimated $\lambda$ leads to a negative mean radial velocity of $-5.6(+11.1,-9.6) \mathrm{km} \mathrm{s}^{-1}$. Thus, although the nominal value of $\lambda$ implies a small degree of expansion, the difference between the observed and geometric radial velocities is within the uncertainty of the latter. It cannot be established from the available data if the association undergoes a general expansion or not. The pattern in Figure 1 is more consistent with two overlapping groups with slightly cris-crossing velocities. In Lupus, a group of 1 Myr old stars around the Lupus 3 dark filament also appears to have slightly different direction of motion from the bulk of older stars, but these clues should be verified with more accurate data. The geometric center of the association lies approximately at ICRS $(-54,-121,-59)$ pc.

Internal dispersion of coordinate velocity is another essential parameter derived from the convergent point analysis. In gravitationally bound, dynamically relaxed open clusters, this parameter is related to the total mass and segregation of massive stars in the center. Collisions and exchange of energy between stars are negligible in associations, and the internal dispersion of velocities is directly related to the conditions and origins of star formation. For example, the dynamical model of star formation (Hartmann et al. 2001) requires the presence of internal flows of gas sporadically merging and dissipating after a short burst of new stars due to the internal turbulent motion. The null results and the tight upper limits on velocity dispersion in the Alpha Persei cluster (Makarov 2006) and the Lupus association (Makarov 2007a) is puzzling, since they imply a small internal turbulent motion of $\lesssim 1 \mathrm{~km} \mathrm{~s}^{-1}$. The situation with the Ophiuchus association is different. Figure $2 a$ depicts the distribution of residual relative angular deviations $\Delta_{i} / \sigma_{\Delta_{i}}$ for the Ophiuchus stars specified in 
TABLE 1

Pre-Main-Sequence Members of the Greater Ophiuchus Association

\begin{tabular}{|c|c|c|c|c|c|c|c|c|}
\hline Number & Name & $\begin{array}{l}\text { R.A. (ICRS) } \\
\quad(\mathrm{J} 2000.0)\end{array}$ & $\begin{array}{l}\text { Decl. (ICRS) } \\
\quad(\mathrm{J} 2000.0)\end{array}$ & $\begin{array}{c}\left(\mu_{\alpha} \cos \delta, \mu_{\delta}\right) \pm\left(\sigma_{\mu_{\alpha *}}, \sigma_{\mu_{\delta}}\right) \\
\left(\operatorname{mas~yr}^{-1}\right)\end{array}$ & $\begin{array}{l}D_{\text {kin }} \\
(\mathrm{pc})\end{array}$ & $\begin{array}{c}J \\
(\mathrm{mag})\end{array}$ & $\begin{array}{c}K_{s} \\
(\mathrm{mag})\end{array}$ & $\begin{array}{c}v_{\perp} \\
\left(\mathrm{km} \mathrm{s}^{-1}\right)\end{array}$ \\
\hline $1 \ldots \ldots \ldots \ldots \ldots \ldots \ldots$ & $\rho \mathrm{Oph}$ & 162535.12 & -232649.8 & $(-5.6,-25.0) \pm(1.2,0.8)$ & 149 & & & -4.1 \\
\hline $2 \ldots \ldots \ldots \ldots \ldots \ldots \ldots$ & HD 147932 & 162535.08 & -232418.8 & $(-8.7,-26.9) \pm(1.1,0.6)$ & 135 & 6.11 & 5.78 & -2.4 \\
\hline 3.................... & WSB 44 & 162702.38 & -230959.2 & $(-26.7,-31.0) \pm(12.4,12.2)$ & 93 & 10.83 & 9.44 & 4.9 \\
\hline $4 \ldots$ & $\mathrm{I}-510$ & 162218.53 & -232148.2 & $(-10.2,-31.3) \pm(4.6,4.8)$ & 116 & 9.515 & 8.109 & -2.6 \\
\hline $5 \ldots$. & HD 147808 & 162451.36 & -223932.6 & $(-14.9,-21.7) \pm(1.8,1.5)$ & 145 & 7.779 & 7.084 & 2.7 \\
\hline ............... & GSC 06794-00337 & 162739.56 & -224523.1 & $(-14.0,-25.0) \pm(1.7,3.4)$ & 133 & 8.942 & 8.084 & 1.2 \\
\hline $7^{*} \ldots \ldots \ldots \ldots \ldots \ldots$ & V 2058 Oph & 162556.17 & -242048.2 & $(-14.1,-17.7) \pm(5.1,5.0)$ & 170 & 9.154 & 7.518 & 4.2 \\
\hline $8 \ldots \ldots \ldots \ldots \ldots \ldots \ldots$ & TYC 6798-544-1 & 162519.25 & -242652.7 & $(-20.5,-26.2) \pm(1.9,1.4)$ & 116 & 8.363 & 7.798 & 4.0 \\
\hline 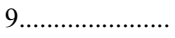 & HD 147196 & 162119.19 & -234228.7 & $(-11.0,-24.2) \pm(0.9,0.6)$ & 144 & 6.57 & 6.42 & -0.5 \\
\hline $10 \ldots \ldots \ldots \ldots \ldots \ldots$ & HD 147889 & 162524.32 & -242756.6 & $(-2.3,-25.5) \pm(1.4,0.9)$ & 150 & 5.34 & 4.58 & -6.7 \\
\hline $11 \ldots \ldots \ldots \ldots \ldots \ldots$ & V 2129 Oph & 162740.27 & -242204.2 & $(-15.5,-33.5) \pm(1.5,1.5)$ & 104 & 8.440 & 7.207 & -0.1 \\
\hline $12 \ldots \ldots \ldots \ldots \ldots \ldots$ & V 852 Oph & 162524.35 & -242944.4 & $(-5.2,-25.4) \pm(5.5,5.0)$ & 148 & 10.748 & 9.446 & -4.4 \\
\hline $13 \ldots$ & HD 147384 & 162236.73 & -242258.7 & $(-3.3,-26.0) \pm(1.2,1.4)$ & 147 & 7.448 & 7.157 & -5.9 \\
\hline $14 \ldots .$. & GSC 06794-00480 & 162045.96 & -234820.9 & $(-14.5,-25.8) \pm(5.0,5.0)$ & 130 & 9.867 & 8.927 & 1.0 \\
\hline $15 \ldots \ldots \ldots \ldots \ldots \ldots$ & HD 147343 & 162219.90 & -242148.2 & $(-9.2,-21.0) \pm(2.1,2.2)$ & 168 & 7.567 & 7.096 & -0.7 \\
\hline $16^{*} \ldots \ldots$ & BKLT J162623-244311 & 162623.70 & -244313.9 & $(-10.4,-23.2) \pm(5.0,5.0)$ & 151 & 9.391 & 7.847 & -0.4 \\
\hline $17^{*} \ldots \ldots \ldots \ldots \ldots$ & BKLT J162644-244312 & 162644.32 & -244313.9 & $(1.9,-18.3) \pm(5.2,5.0)$ & 209 & 10.989 & 9.573 & -9.9 \\
\hline $18 \ldots \ldots$. & HD 147283 & 162157.69 & -242943.5 & $(-14.7,-23.1) \pm(1.0,1.5)$ & 141 & 8.124 & 7.587 & 2.1 \\
\hline $19^{*} \ldots \ldots$ & BKLT J162752-244049 & 162752.08 & -244050.4 & $(-12.0,-17.1) \pm(5.3,5.3)$ & 184 & 9.997 & 8.126 & 3.2 \\
\hline $20 \ldots \ldots \ldots \ldots \ldots \ldots \ldots \ldots \ldots \ldots$ & HD 147137 & 162050.23 & -223538.8 & $(-8.0,-18.9) \pm(1.1,1.3)$ & 186 & 8.032 & 7.785 & -1.1 \\
\hline 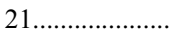 & HBC 644 & 163104.36 & -240433.4 & $(-14.2,-28.6) \pm(3.0,2.9)$ & 120 & 8.861 & 7.784 & 0.6 \\
\hline $22 \ldots$ & HD 147220 & 162121.14 & -220632.2 & $(-21.4,-17.3) \pm(1.3,1.2)$ & 138 & 7.619 & 7.372 & 7.7 \\
\hline $23^{*}$. & WSB 71 & 163130.88 & -242439.9 & $(-18.9,-31.0) \pm(5.1,5.0)$ & 105 & 10.827 & 7.900 & 2.2 \\
\hline $24^{*}$. & ROXs 43A & 163120.12 & -243005.1 & $(-5.9,-29.2) \pm(3.3,1.6)$ & 129 & 7.904 & 6.729 & -4.1 \\
\hline $25 \ldots \ldots$ & GSC 06214-02384 & 161933.96 & -222829.4 & $(-18.0,-25.9) \pm(2.9,2.4)$ & 121 & 9.230 & 8.509 & 2.6 \\
\hline $26 \ldots \ldots \ldots \ldots \ldots \ldots$ & V 2394 Oph & 163140.68 & -242516.2 & $(-6.7,-24.5) \pm(1.1,1.3)$ & 151 & 7.531 & 6.787 & -2.9 \\
\hline $27^{*} \ldots$ & WSB 72 & 163133.46 & -242737.2 & $(-13.3,-15.6) \pm(5.0,5.0)$ & 187 & 9.233 & 7.610 & 5.1 \\
\hline $28^{*} \ldots \ldots \ldots \ldots \ldots$ & ROXs 42C & 163115.75 & -243402.2 & $(-10.6,-19.2) \pm(3.1,2.9)$ & 175 & 8.357 & 7.129 & 1.4 \\
\hline $29 \ldots \ldots$. & PDS 415 & 161837.24 & -240522.6 & $(-10.9,-27.3) \pm(4.2,1.9)$ & 131 & 9.020 & 7.899 & -1.5 \\
\hline $30 \ldots \ldots \ldots$ & HD 148562 & 162954.60 & -245846.1 & $(-13.7,-26.4) \pm(1.1,0.6)$ & 129 & 7.46 & 7.31 & 0.9 \\
\hline $31 \ldots \ldots \ldots \ldots \ldots \ldots$ & V 2505 Oph & 162948.69 & -215211.9 & $(-5.6,-22.1) \pm(3.6,1.8)$ & 166 & 8.677 & 7.756 & -3.4 \\
\hline $32 \ldots \ldots$ & HD 146516 & 161731.39 & -230336.1 & $(-13.2,-17.3) \pm(1.2,1.4)$ & 176 & 8.514 & 7.967 & 3.3 \\
\hline $33^{*} \ldots$ & V 2248 Oph & 163211.80 & -244021.6 & $(-12.2,-14.4) \pm(5.0,5.0)$ & 203 & 9.245 & 7.929 & 5.1 \\
\hline $34 \ldots \ldots \ldots$ & i Sco & 163012.48 & -250654.8 & $(-3.4,-26.5) \pm(0.9,0.5)$ & 144 & 5.03 & 5.09 & -5.5 \\
\hline $35 \ldots \ldots \ldots \ldots \ldots \ldots$ & HD 147648 & 162402.89 & -252454.0 & $(-11.9,-25.1) \pm(1.1,1.2)$ & 139 & 7.289 & 6.719 & -0.1 \\
\hline $36 \ldots \ldots \ldots \ldots \ldots \ldots$ & LSE 96 & 162332.33 & -252348.6 & $(-15.9,-23.1) \pm(1.6,2.7)$ & 138 & 8.600 & 7.695 & 2.8 \\
\hline 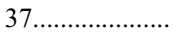 & HD 146367 & 161653.38 & -230637.7 & $(-7.8,-24.4) \pm(1.1,1.2)$ & 150 & 7.636 & 7.336 & -2.9 \\
\hline $38 \ldots \ldots$ & TYC 6793-1406-1 & 161617.95 & -233947.6 & $(-8.7,-26.1) \pm(2.0,1.7)$ & 140 & 8.727 & 8.102 & -2.7 \\
\hline 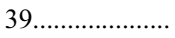 & VV Sco & 161534.57 & -224242.5 & $(-26.3,-51.3) \pm(12.6,12.9)$ & 66 & 9.382 & 7.909 & -0.0 \\
\hline 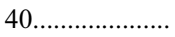 & HD 146743 & 161839.14 & -213534.2 & $(-11.1,-24.4) \pm(1.2,1.1)$ & 142 & 7.838 & 7.542 & -0.8 \\
\hline 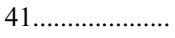 & HD 146897 & 161929.24 & -212413.3 & $(-11.9,-25.6) \pm(1.3,1.2)$ & 134 & 8.062 & 7.800 & -0.6 \\
\hline $42 \ldots \ldots \ldots \ldots \ldots \ldots$ & HD 147013 & 162015.04 & -253844.4 & $(-12.1,-22.0) \pm(1.1,1.2)$ & 154 & 7.936 & 7.667 & 0.9 \\
\hline $43 \ldots \ldots$. & HD 146285 & 161625.17 & -245919.5 & $(-14.9,-25.2) \pm(1.2,1.3)$ & 132 & 7.240 & 7.025 & 1.2 \\
\hline $44 \ldots \ldots \ldots \ldots \ldots \ldots$ & TYC 6793-819-1 & 161411.08 & -230536.2 & $(-12.1,-23.8) \pm(1.6,1.9)$ & 144 & 8.280 & 7.458 & -0.2 \\
\hline $45 \ldots \ldots \ldots \ldots \ldots \ldots$ & HD 147255 & 162147.93 & -255647.2 & $(-8.0,-24.0) \pm(1.5,1.1)$ & 153 & 8.292 & 8.029 & -2.4 \\
\hline 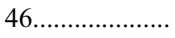 & GSC 06793-00994 & 161402.12 & -230102.2 & $(-8.8,-22.8) \pm(1.7,1.7)$ & 157 & 9.375 & 8.608 & -2.0 \\
\hline $47 \ldots \ldots$ & GSC 06793-00569 & 161329.29 & -231107.6 & $(-12.4,-30.8) \pm(2.0,2.5)$ & 116 & 9.317 & 8.494 & -1.8 \\
\hline $48 \ldots \ldots \ldots \ldots \ldots \ldots$ & HD 147592 & 162347.17 & -261615.8 & $(-11.6,-21.1) \pm(1.1,1.1)$ & 161 & 8.152 & 7.972 & 1.1 \\
\hline 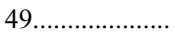 & GSC 06214-00210 & 162154.67 & -204309.1 & $(-19.5,-29.8) \pm(4.0,4.2)$ & 106 & 9.998 & 9.152 & 2.1 \\
\hline $50 \ldots \ldots \ldots \ldots \ldots \ldots$ & V 1051 Sco & 161345.50 & -242519.5 & $(-10.0,-19.2) \pm(0.9,0.7)$ & 178 & 6.16 & 6.08 & 0.1 \\
\hline $51 \ldots \ldots \ldots \ldots \ldots \ldots$ & V 895 Sco & 162134.71 & -261226.9 & $(-9.6,-18.4) \pm(5.0,5.0)$ & 187 & 10.096 & 8.864 & 0.5 \\
\hline $52 \ldots \ldots \ldots \ldots \ldots \ldots$ & GSC $06793-00797$ & 161302.72 & -225744.5 & $(-12.7,-23.3) \pm(3.2,3.3)$ & 145 & 9.322 & 8.455 & 0.3 \\
\hline $53 \ldots \ldots \ldots \ldots \ldots \ldots$ & HD 146416 & 161658.77 & -211814.9 & $(-14.3,-25.4) \pm(1.0,0.7)$ & 130 & 6.51 & 6.49 & 0.7 \\
\hline 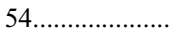 & StHA 124 & 161301.30 & -240654.7 & $(-6.5,-23.5) \pm(4.6,4.6)$ & 158 & 9.667 & 8.752 & -3.8 \\
\hline $55^{*} \ldots \ldots \ldots \ldots \ldots \ldots$ & GSC 06214-00039 & 161853.83 & -205318.2 & $(-7.6,-34.5) \pm(12.3,12.3)$ & 107 & 8.645 & 7.213 & -4.6 \\
\hline $56^{*} \ldots \ldots \ldots \ldots \ldots$ & WSB 82 & 163945.44 & -240203.9 & $(-10.9,-22.3) \pm(4.9,4.9)$ & 153 & 10.174 & 7.629 & 0.9 \\
\hline $57^{*} \ldots \ldots \ldots \ldots \ldots$ & V 2307 Oph & 164017.92 & -235345.2 & $(-6.2,-18.9) \pm(1.1,1.3)$ & 191 & 6.947 & 5.476 & -1.5 \\
\hline $58 \ldots \ldots \ldots \ldots \ldots \ldots$ & TYC $6813-143-1$ & 164526.16 & -250316.7 & $(-2.9,-24.5) \pm(1.5,2.4)$ & 155 & 8.794 & 7.725 & -4.8 \\
\hline
\end{tabular}

Notes._- Stars whose location on the near-infrared HR diagram suggests they may be as young as 1 Myr are marked with asterisks in the first column. Units of right ascension are hours, minutes, and seconds, and units of declination are degrees, arcminutes, and arcseconds. 


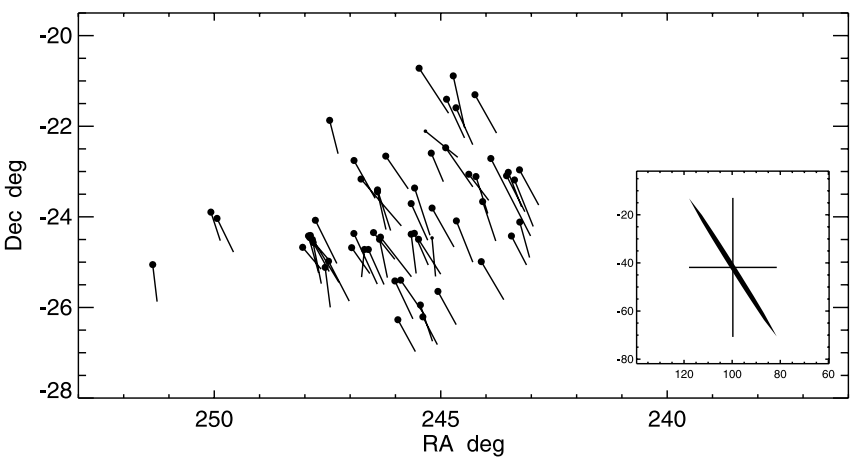

FIG. 1.- ICRS positions and proper motions of pre-main-sequence stars in the greater Ophiuchus T-association. Typical length of proper motion vectors is $27 \mathrm{mas}^{-1}$. The inset shows the convergent point of the association (in the crosshairs), the $1 \sigma$ error ellipse and the projected coordinate error bars, as geometrically determined by the Amoeba algorithm.

Table 1 . The parameters $\Delta_{i}$ are the angular deviations of individual proper motion vectors from the great circle connecting the star with the convergent point, and $\sigma_{\Delta_{i}}$ are their expected standard deviations. The latter are the quadratic sum of the astrometric angular error directly computed from the proper motion errors, and the physical one-dimensional dispersion. The physical dispersion term is iteratively adjusted until the fitting Gaussian, shown with the thin line in the graph, reaches a $\sigma=1$. A physical dispersion of $2 \mathrm{~km} \mathrm{~s}^{-1}$ is obtained in this manner for the Ophiuchus stars. This term becomes the dominating contribution to the total $\sigma_{\Delta_{i}}$ for more than half of the sample. Figure $2 b$ depicts the histogram of the transverse tangential velocity components $v_{\perp}$ (Makarov \& Robichon 2001), which are orthogonal to the great circles connecting the stars and the convergent point, and lie in the plane of the sky. We have determined that this distribution includes a physical scatter of $\sigma_{\text {intrinsic }}=2 \mathrm{~km} \mathrm{~s}^{-1}$. The Gaussian fit of the histogram has a standard deviation of $3.1 \mathrm{~km} \mathrm{~s}^{-1}$. In the event that the distribution may be non-Gaussian, an alternative estimation of the observed dispersion as the half-difference between the 84 th and 16th percentiles yields $3.2 \mathrm{~km} \mathrm{~s}^{-1}$. This value is the robust upper limit on the one-dimensional velocity dispersion as observed, with the most likely value at $2 \mathrm{~km} \mathrm{~s}^{-1}$.

The histogram of heliocentric distances to Ophiuchus stars is shown in Figure $2 c$. The median distance, $145 \mathrm{pc}$, is the same as the mean distance. The interquartiles of the distribution are 130, 145 , and $158 \mathrm{pc}$; therefore, half of the members lie within $\simeq 15 \mathrm{pc}$ of the center. Since some of the stars have quite uncertain kine- matic distances, the size of the association is conservatively assumed to be $30 \mathrm{pc}$.

\section{AGES AND STAR FORMATION HISTORY}

Significant evidence has been found that new stars are generated in nearby star-forming regions over time spans of 10-30 Myr rather than in single bursts. Some probable members of the Orion nebula cluster are $10 \mathrm{Myr}$ old, as defined by depletion of lithium in their atmospheres (Palla et al. 2005). The first generation of Lupus pre-main-sequence stars was probably formed $28 \mathrm{Myr}$ ago (Makarov 2007a). The observed spread of isochrones for Ophiuchus suggests an upper range of $10 \mathrm{Myr}$ (Bouvier \& Appenzeller 1992; Wilking et al. 2005) for stars outside the main dark nebula. Using the distances to all members listed in Table 1, I present a $M_{K s}-\left(J-K_{S}\right)$ diagram of the dispersed Ophiuchus population in Figure 3. The near-infrared magnitudes from 2MASS are dereddened by a general $A_{V}=1 \mathrm{mag}$. The 14, 5, 1 and $0.1 \mathrm{Myr}$ isochrones from (Siess et al. 2000) are shown for comparison with thin broken lines. The diagram displays a large spread in $J-K_{s}$ color. Two of the members, WSB 71 (star 23) and WSB 82 (star 56) fall outside the plotted area, with observed $J-K_{s}=$ 2.93 and $2.54 \mathrm{mag}$, respectively. Other abnormal infrared stars are marked with crosses in the graph, and also flagged with asterisks in Table 1. Obviously, Siess et al. models fail to reproduce the reddest colors in Ophiuchus. But the $14 \mathrm{Myr}$ isochrone seem to outline well the blue boundary of the distribution.

There are two significant sources of apparent reddening in Ophiuchus. Because of the fractal structure of the complex, the presence of a dense nebulae, and the spread in distances, a considerable degree of differential extinction can be expected for this large area. The long inclined arrow in Figure 3 indicates the expected reddening at $A_{V}=5 \mathrm{mag}$. Differential reddening moves the data points to the right (redder) and down (fainter). Since all our stars are outside the main dark cloud, a differential extinction larger than 5 mag is unlikely. In the absence of spectroscopic data for most of the stars, individual extinction values can only be guessed from secondary information. For a few stars in our sample, interstellar extinction is estimated in (Wilking et al. 2005), namely, stars 7 ( $\left.A_{v}=0.5 \mathrm{mag}\right), 11(0.0), 12(0.0), 16(2.9), 17$ (2.4), and 19 (5.0). The infrared stars in this group are 7, 16, 17, and 19. As all the stars in the H-R diagram have already been dereddened by $A_{V}=1 \mathrm{mag}$, these corrections do not go far in bringing the colors into the range of theoretical models, even for the star 19 (BKLT J162752-244049) with the largest spectroscopic reddening. We note that some of the infrared stars appear
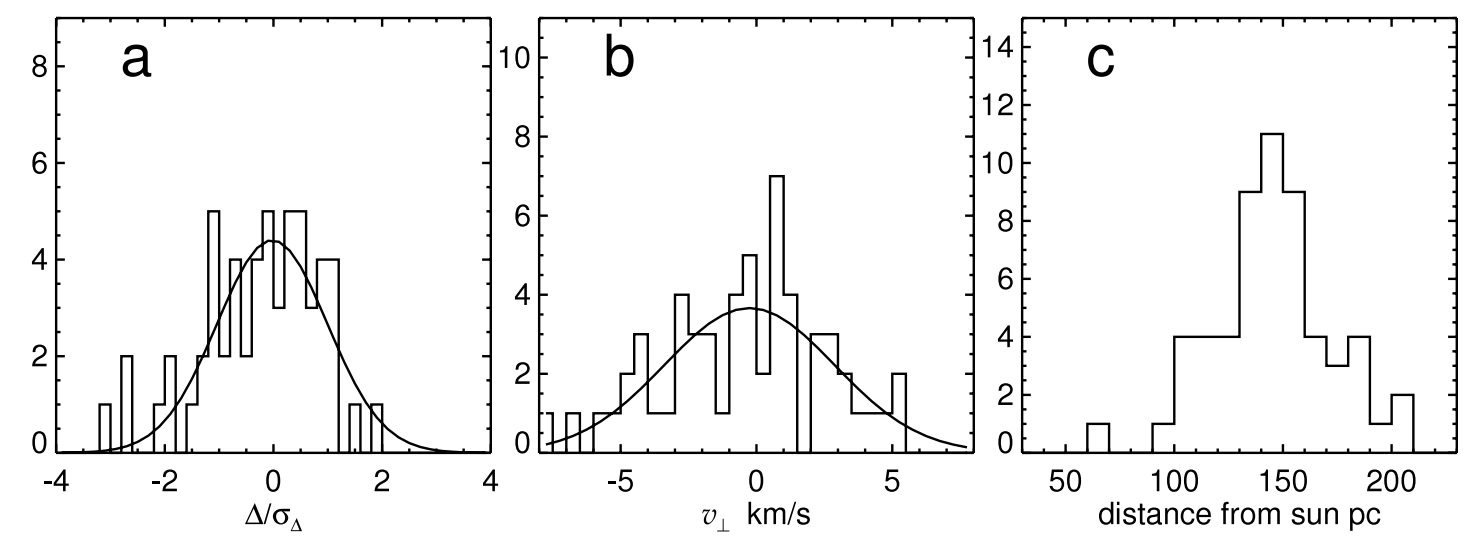

FIG. 2.-Distributions, from left to right, of $(a)$ relative angular deviations of proper motion vectors from the common convergent point, $(b)$ absolute transverse tangential velocities in $\mathrm{km} \mathrm{s}^{-1}$, and $(c)$ distances from the Sun in parsecs. Gaussian fits are shown for the former two histograms. 


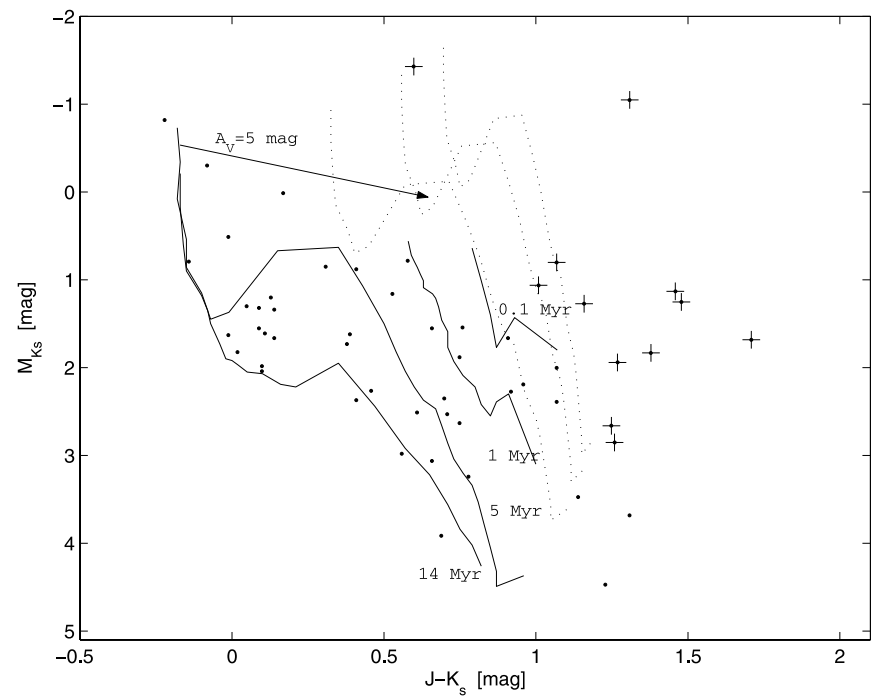

FIG. 3.- Near-infrared H-R diagram of Ophiuchus with individual distances obtained by the moving cluster method (see Table 1 and text). Four isochrones $(14,5,1$ and $0.1 \mathrm{Myr}$ ) are drawn from (Siess et al. 2000) at $Z=0.01$ and general $A_{V}=1 \mathrm{mag}$. The $5 \mathrm{Myr}$ isochrone is drawn for the cases when CTTSs with accretion disks have near infrared excesses from the inner rim heated to $T_{\text {eff }}=$ $1750 \mathrm{~K}$ of 1,2 , and 3 times the stellar photospheric flux in $K_{s}$. Stars supposed to be the youngest in the association are marked with plus signs.

to be significantly more distant than the center, for example, the remarkably bright and red object V2307 Oph (star 57) with a kinematic distance of $191 \mathrm{pc}$ and a small formal error. Such members have better chances to be obscured by foreground interstellar medium.

The second reason for young stars to be redder than expected is a nonphotospheric radiation in the near infrared. Cieza et al. (2005) present substantial evidence that CTTSs often display intrinsic excess in the $J$ and $K$ passbands. These stars have massive accreting disks radiating in the mid-infrared, and the near infrared excess is interpreted as the light emitted by the incandesced inner rims of the disks. High-resolution interferometric observations confirm the existence of circumstellar disks of large inner radii (Colavita et al. 2003) required to generate a measurable $J$ and $K$ excess. If the excess flux in $K$ is

$$
F_{K_{\mathrm{ex}}}=f F_{K_{0}},
$$

where $F_{K_{0}}$ is the photospheric $K$-band flux, and $\alpha_{J K}$ is the spectral index of the excess flux in $J$ and $K$,

$$
F_{K_{\mathrm{ex}}}=\alpha_{J K} F_{J_{\mathrm{ex}}}
$$

then the observed magnitudes are

$$
\begin{aligned}
K_{\mathrm{obs}}= & -2.5 \log F_{K_{0}}-2.5 \log (1+f), \\
& J_{\mathrm{obs}}-K_{\mathrm{obs}}=-2.5 \log \left(F_{J_{0}}+\frac{f}{\alpha_{J K}} F_{K_{0}}\right) \\
& +2.5 \log F_{K_{0}}+2.5 \log (1+f) .
\end{aligned}
$$

The empirical $\alpha_{J K} \approx 3.2$ (Cieza et al. 2005), which corresponds to blackbody radiation at $T_{\text {eff }}=1750 \mathrm{~K}$, the sublimation point of interstellar dust.

The 5 Myr isochrone in Figure 3 is shown for $f=1,2$ and 3, as indicated with dotted lines. The loci of stars with powerful disks are displaced to redder colors and to brighter absolute $K_{s}$ magnitudes. The range $f \in[0,3]$ is perhaps what can be reason-

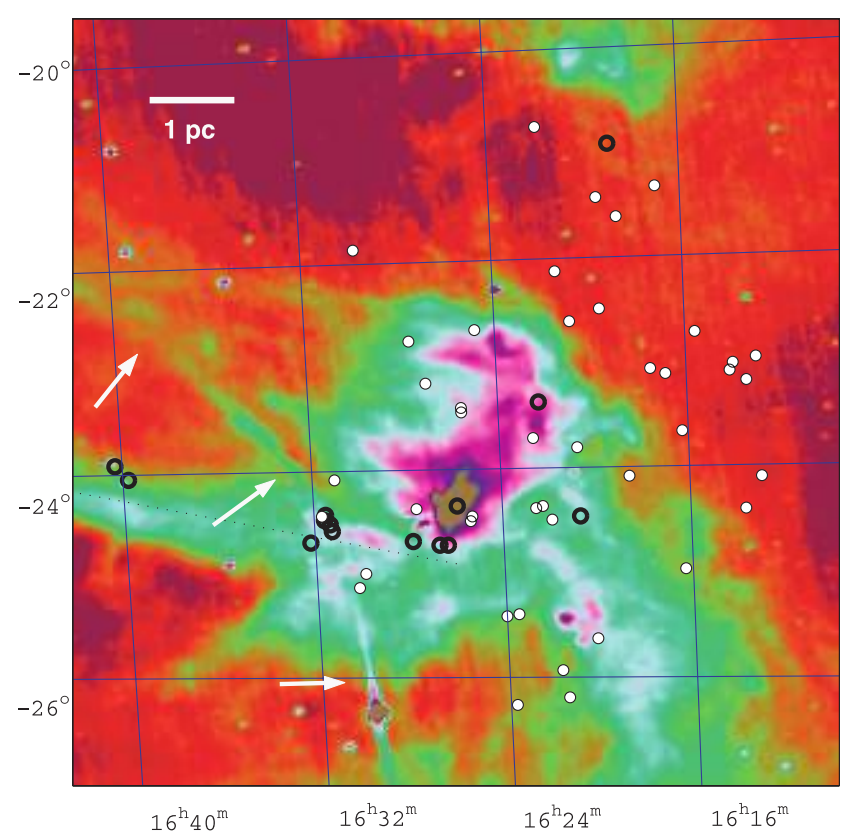

FIG. 4.-IRAS $12 \mu \mathrm{m}$ map of the cloud complex around $\rho$ Oph with our sample stars overlaid. The general stars are marked with small white-colored circles, but the probable youngest stars selected from the near-infrared HR diagram in Fig are marked with larger, thickly outlined circles. Note the alignment of the youngest stars with the broad filament (Streamer) extending west to east and originating just south of the densest part of the cloud, indicated with the dotted black line. Also note the small cluster of young stars at the intersection of this filament with the narrow arc (marked with white arrows) at $\sim\left(16^{\mathrm{h}} 30^{\mathrm{m}},-24.5^{\circ}\right)$, called the ROX 43 cluster. The physical scale of this map at the mean distance to the association is indicated in the top left corner.

ably expected in very young CTTSs; it becomes obvious that only some, not-so-red stars can be explained by the admixture of near-IR radiation from the disks. The nonphotospheric nearinfrared radiation from young stars is expected to be correlated with the mid-infrared fluxes from IRAS. In order to find out which stars specifically may have enhanced levels of disk nearIR radiation, we consider the mid-infrared colors from IRAS.

It is possible to roughly differentiate pre-main-sequence stars at different stages of evolution and deeply embedded sources in the IRAS color-color diagrams (Beichman et al. 1986; Adams et al. 1988; Weintraub 1990). Eight out of 13 selected infrared stars are powerful IRAS sources, indicating that the disk radiation may indeed make a significant contribution to the joint photometry. These eight stars are 7 (IRAS 16229-2413), 23 (162842418), 24 (16283-2423), 27 (16285-2421), 28 (16282-2427), 56 (16367-2356), and 57 (16372-2347). The star V2307 Oph (star 57) is the most powerful mid-infrared source, clearly seen on the IRAS map (Fig. 4) as well as on the high-resolution MIPS map from Spitzer (Fig. 5). This star has unusual IRAS colors compared to the well-defined range of T Tauri stars (e.g., Beichman et al. 1986), with $[25 / 12]=0.01$ straddling the lower limit, and $[60 / 25]=-0.35$ being actually slightly below the lower boundary. This is a hint at a considerable short-wave excess extending to the near-infrared. There are other near-infrared stars close to the lower boundary in the [25/12] color, viz., stars 23 (0.09), and $55(-0.01)$.

Interestingly, two of the stars with low estimated extinction from Wilking et al. (2005), 7 and 11, are extremely powerful IRAS sources at $100 \mu \mathrm{m}$. These two T Tauri stars are projected against the relatively sparse cloud of warm dust roughly centered on star 7 (see Fig. 4), where most of the nebulous IRAS sources reside. 


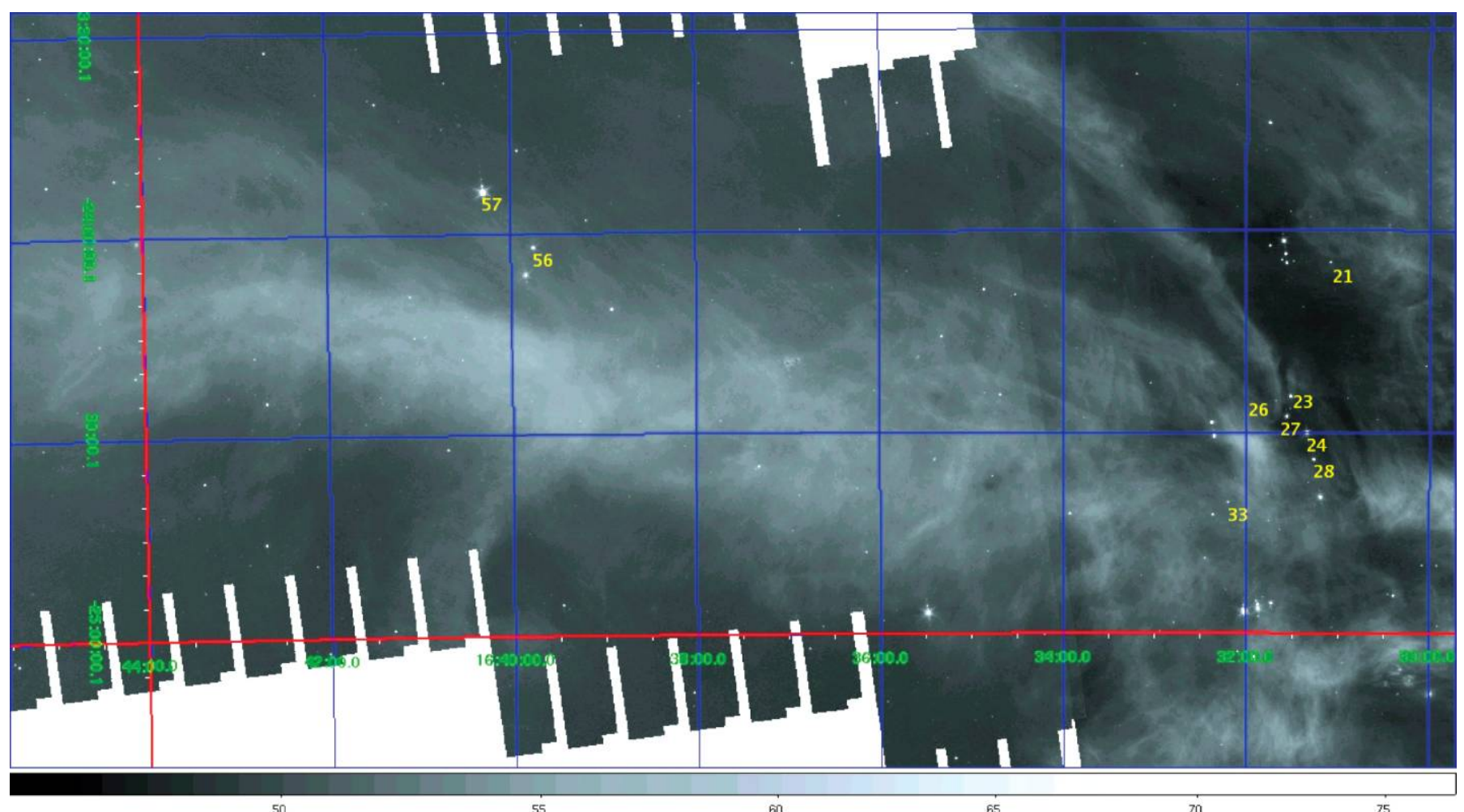

FIG. 5. - High-resolution map of the Ophiuchus Streamer area (clouds L1689, L1712, and L1729) at $24 \mu$ m obtained with Spitzer MIPS as part of the c2d Legacy program. Astrometric members of the Ophiuchus association are marked with their numbers from Table 1 in yellow.

They both have measurable $\mathrm{H} \alpha$ emission, especially star 7 (Wilking et al. 1987). The three stars of the largest estimated extinction, stars 16,17 , and 19 , on the contrary, lie outside the warm dusty cloud lining up along its southern extent. This segregation may be related to the fact that the extended cloud of warm dust (the IRAS cloud), also visible as the slightly glowing background on the combined color-coded map from the $2 \mathrm{MASS}$ calibration images in Figure 6, is offset with respect to the dense

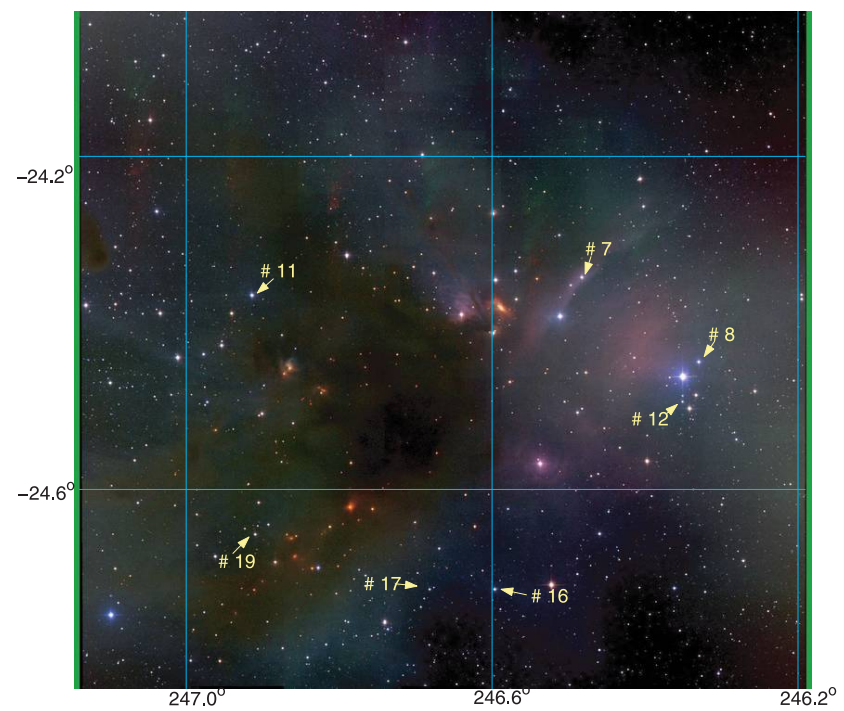

FIG. 6.-Combined 2MASS calibration map of the central Ophiuchus cloud. The sample stars discussed in this paper are marked with their running numbers from Table 1 and arrows. Note the star V2058 Oph (star 7) situated in the middle the IRAS core of warm dust is actually outside the dense and cold cloud at $\left(16^{\mathrm{h}} 27^{\mathrm{m}},-24.5^{\circ}\right)$. and cold core in the west direction. Figure 7 shows more clearly that the bulk of the filamentary and clumpy cloud of hot dust visible on the mosaic Spitzer MIPS image at $24 \mu \mathrm{m}$ does not match positionally the densest cores of cold molecular material indicated with intensity contours from the ${ }^{12} \mathrm{CO}$ line map in (Ridge et al. 2006). One of the two densest CO cores (marked with magenta crosses in the plot) is coincident with a gap separating an entwined filament and a cometary clump of hot dust. The small clusters of young stellar objects are better aligned with the molecular features than with the hot dust, as expected. I conclude from this analysis that even after appropriate corrections for possible extinction in the outskirts of the Ophiuchus cloud and for excess emission from the inner parts of optically thick disks, the extreme near-infrared stars appear to be very young, probably $\sim 1$ Myr or younger.

\section{STAR FORMATION OUTSIDE THE MAIN CORE}

Assuming that the intrinsic velocity dispersion of $2 \mathrm{~km} \mathrm{~s}^{-1}$ per coordinate direction has been uniform over the time span of star formation, the older members (10-14 Myr) could have dispersed out to 20-30 pc from their birth places and fill in the volume observed today. On the other hand, it is hardly possible that the main cloud and the embedded cluster in it are responsible for the existence of these stars. According to the dynamical model of star formation in molecular clouds (Ballesteros-Paredes et al. 1999; Hartmann et al. 2001), such high-density cores are transient formations, which cannot survive and sustain star formation for longer than a few Myr. The older stars were born outside the current main cloud in separate cores that have dissipated or blended into other extended features. The center of the older population appears to be offset in the northwest direction from the concentration of warm dust (Fig. 4) and from the cold dark cloud (Fig. 6). The center of activity has moved by roughly $5 \mathrm{pc}$ in the 


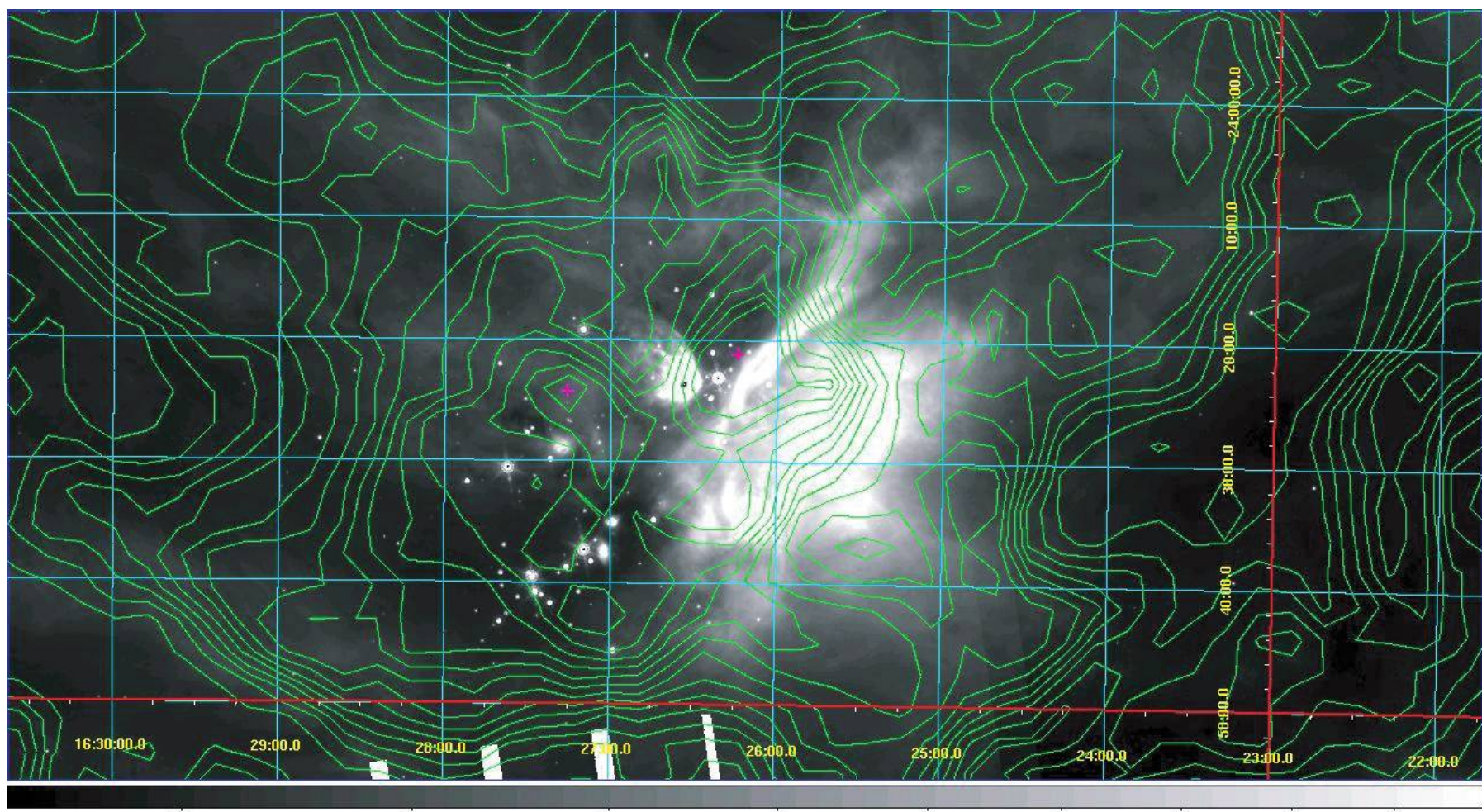

50

60

70

100

110

FIG. 7.-Spitzer MIPS $24 \mu \mathrm{m}$ map of the central Ophiuchus area (cloud L1688), overlaid with intensity contours from the ${ }^{12}$ CO map from (Ridge et al. 2006). The

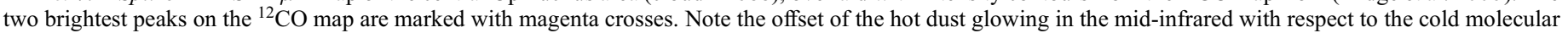
clumps, and a better alignment of young stellar objects with the molecular material.

sky projection, in about 10 Myr. The youngest stars, especially those younger than $1 \mathrm{Myr}$, could not have moved far from their birth sites. They are marked with bigger black circles in Fig. 4. It is evident that most of these stars are located along a relatively narrow strip closely following the eastern streamer of warm dust, indicated with a dotted black line in this plot. These stars were recently formed in this extended structure stretching over $8 \mathrm{pc}$. A more detailed view of the eastern streamer is provided in Figure 5, which is a reproduction of the mosaic $24 \mu \mathrm{m}$ MIPS map obtained with Spitzer (Evans et al. 2003).

Figure 6 displays more clearly the central part of the association and the main dark cloud. Three of the four presumably youngest stars present in the map, stars 16,17 , and 19, are clearly offset from the densest regions of the cloud to the south. The estimated extinction for these stars is $A_{V}=2.9,2.4$, and $5.0 \mathrm{mag}$, respectively. The remarkable star V2058 Oph (star 7) is situated on the other side of the main cloud, and outside the filament. This star is projected against a bright, sharply outlined swath on the 2MASS image, corresponding to the photodissociation front nurtured by the young B2 star HD 147889 (Habart et al. 2003). There is also a compact dark globule just to the north of V2058 Oph, clearly seen on blue photographic finder charts, for example. A kinematic distance of $170 \mathrm{pc}$ (Table 1) implies that this star can be strongly reddened.

Apart from the three stars to the south of the main cloud, the eastern filament is outlined by a compact group around the star ROXs 43a (star 24) and two remote objects in the tip, stars 56 and 57 , both outstandingly bright $I R A S$ sources. The true extent and the filamentary morphology of the streamer are better seen in Figure 5 and on the 2MASS extinction maps, and ${ }^{12} \mathrm{CO}$ and ${ }^{13} \mathrm{CO}$ line maps in (Ridge et al. 2006). De Geus et al. (1990) suggested that the filamentary morphology of the $\rho$ Oph complex was gen- erated by several supernova explosions in the adjoining Upper Scorpius OB association. If it was a supernova that created the narrow circular segment (indicated with arrows in Fig. 4), it exploded on the opposite side of the cloud from Upper Scorpius. The origin of the broad eastern streamer is a matter of dispute, with penetrating shock waves from a different supernova explosion to the north and west of the cloud being one of the suggested explanations (de Geus et al. 1990). However, this feature is clumpy, much more diffuse than the circular segment, and has a small curvature. An alternative explanation for such nearly linear filaments of gas and dust is two large, diffuse gas streams merging at moderate relative velocities. This may be a common phenomenon, since similar extended filaments outlined by concentrated distributions of Class II infrared objects are found in other starforming regions, for example, in the $\lambda$ Orionis Cluster (Barrado y Navascués et al. 2007). Three-color images of the eastern streamer from Spitzer (Young et al. 2006, their Fig. 5) show a pronounced sidewise segregation of the filament in density and temperature, with the northern edge sharply outlined by emission in the longer $160 \mu \mathrm{m}$ wavelength.

The ROXs 43 group is suggestively coincident with the intersection of the eastern filament and a very narrow arc of warm dust clearly visible in the IRAS map (Fig. 4, three white arrows). A closer look at the area reveals that the four stars with extreme infrared colors, stars 23, 24, 27, and 28, are the brightest objects in a minicluster counting more than a dozen stars. ${ }^{1}$ Few of them have been investigated yet, but the 2MASS catalog lists some very red stars in an area of $13^{\prime}$ radius centered on ROXs $43 \mathrm{~A}$. A few stars in the ROX 43 cluster, including WSB 71 (star 23)

The Aladin web-based application maintained at CDS provides a highquality SERC photographic image of the area. 
appear to be nonhierarchical multiple systems, which are inherently unstable.

\section{A SEARCH FOR STARS EJECTED FROM OPHIUCHUS}

Numerical simulations of dense, rich open clusters of the Orion Nebula type (ONC) suggest that a large fraction of solartype and low-mass members is ejected at escape velocities during the initial dynamical relaxation, after the bulk of gas has been swept out by the first massive stars. The ejected members follow their individual galactic orbits, drifting away from the parent cluster, and some are bound to end up in the vicinity of the Sun, if the cluster is not too distant. Finding such stars would provide insight in the dynamical environment of the past star formation episodes in Ophiuchus, and identify convenient targets for the ongoing study of dusty disks orbiting young stars. I carried out an extensive search for nearby stars that may have been ejected from the association during the past 14 Myr by back-tracking Galactic orbits of all solar type stars from the Geneva-Copenhagen spectroscopic survey (Nordström et al. 2004) and about 200 other stars with accurate radial velocities collected from the literature. The technique of orbit back-tracking is described in previous papers (e.g., Makarov et al. 2004). The number of stars included in this computation is about 9300 . The following mean parameters are adopted for the greater Ophiuchus association: galactic coordinates $(l, b)=\left(353.0^{\circ}, 17.6^{\circ}\right)$, proper motion $\left(\mu_{l} \cos b, \mu_{b}\right)=$

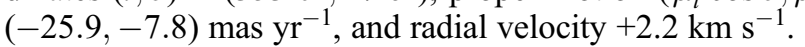

All together, about 50 recent encounters and close fly-bys are identified. The rate of occurrences on the general sample $(>0.5 \%)$ is remarkably high, because similar calculations for other clusters and associations, as well as for random test points, produce significantly smaller numbers. Most likely, the reason for this is the presence of the Hyades stream (or supercluster) of field dwarfs and giants in the local part of the Galaxy (Eggen 1958). The Ophiuchus region is located close to the direction of the Galactic center and slightly above the plane. The Hyades stream has a considerable outward radial motion and a smaller downward motion (Famaey et al. 2005). Thus, the Ophiuchus cloud just happened to be in the way of the Hyades stream, which is a dynamically induced stream of unrelated field stars according to new evidence (Famaey et al. 2005). Indeed, most of the stars from the Geneva-Copenhagen survey, involved in these encounters, are common poorly known stars about which almost nothing can be found in the literature. An interesting possibility presents itself to interpret the relatively high metal abundance of the Hyades supercluster giants as surface pollution at highvelocity passages through the dusty clouds.

Several of the stars that likely flew through parts of the Ophiuchus cloud in the last few Myr are quite interesting, including the candidate planet host HD 89307, two stars with prominent debris disks HIP 43726 and HIP 76829, and the active M0.5 dwarf DT Vir with an astrometric binary companion. All these passages are definitely chance events, since the stars are much older than the age of the Ophiuchus association and the time of encounter, and the fly-by velocities range between 15 and $40 \mathrm{~km} \mathrm{~s}^{-1}$. Only three stars in this collection show signs of youth, the wide visual double HIP 60831 and 60832, and the pre-mainsequence star GJ 182 at 27 pc. The pair HIP 60831 and 60832, although chromospherically active (Strassmeier et al. 2000), have moderate abundances of photospheric lithium, and are probably much older than the upper age envelope of Ophiuchus (14 Myr). The young star GJ 182 deserves special attention, because its evolutionary status is not known. It passed the cloud $6 \mathrm{Myr}$ ago at a distance of $18 \mathrm{pc}$ from the geometric center and a relative velocity of $27 \mathrm{~km} \mathrm{~s}^{-1}$. The high relative velocity implies a chance encounter, because only rather exotic phenomena, such as disruption of binaries by a supernova explosion, can impart a high kinetic momentum on the ejecta. Although credible high-velocity ejections from $\mathrm{OB}$ associations and massive clusters have been identified, including high-velocity ejections from NGC 7023 (Goodman \& Arce 2004) and the Trapezium cluster in Orion (Tan 2004), it seems improbable that the only young star in our sample coming from Ophiuchus belongs to this category. Furthermore, the somewhat conflicting age estimates by isochrone placement (40-50 Myr) and via the surface lithium abundance $(\approx 20 \mathrm{Myr})$ are consistently higher than the time of encounter (Favata et al. 1998). Similar fairly distant fly-bys with the Ophiuchus star formation region (SFR) were found for the young nearby groups TWA and Beta Pictoris, 7 and 11 Myr ago, respectively (Makarov 2007b). The fly-by velocities were substantial in these cases too $\left(\approx 15 \mathrm{~km} \mathrm{~s}^{-1}\right)$, mostly because of the counterdirected heliocentric motion of the Ophiuchus SFR. It was suggested that the TWA and Beta Pictoris progenitor clouds were parts of a large, dispersed complex, which was involved in a grazing encounter with the Ophiuchus complex. The TWA members (or some of them) could be generated in this encounter, but some of the Beta Pic group had already been formed at a different location, possibly in the previous interaction with the R CrA starforming region $20 \mathrm{Myr}$ ago. The enigmatic young star GJ 182 may be a stray member of the larger TWA-BETAPIC stream.

The results of this study indicate that the immediate solar neighborhood (within $\simeq 100 \mathrm{pc}$ ) contains few, if any, young stars dynamically ejected from the Ophiuchus SFR. Thus, a massive isotropic ejection of stars, predicted in the ONC scenario, is unlikely to have taken place in Ophiuchus during the initial star formation episodes. The general expansion and kinematical scatter has been too slow for the first-generation stars to reach the vicinity of the Sun. The dynamical condition of the expanded association is consistent with initial star formation bursts taking place in extended, low-density regions, as opposed to the ONC model. This precluded formation of hard massive binaries and dynamical interaction between newborn systems. The current ONC-like burst in the thickness of the main cloud is the climax of a protracted, large-scale star formation event.

\section{DISCUSSION}

A few stars listed in Table 1 as members of the greater Ophiuchus association have been selected as possible Upper Scorpius (US) OB association members in previous papers (e.g., Preibisch \& Zinnecker 1999). The US is adjoining Ophiuchus at its northwest corner, and there is no clear gap between the associations in the sky plane projection. Furthermore, the systemic velocities of US and Ophiuchus are very similar (Sartori et al. 2003; Makarov $2007 b$ ). On the grand scale, the regions of recent and current star formation in the fourth Galactic quadrant in Ophiuchus, Scorpius, Centaurus, Corona Australis, Lupus, Chamaeleon, Crux, and Carina, appear to form a single supercomplex of young stars, all having similar space velocities, ages between 0 and $30 \mathrm{Myr}$, and distances between 100 and $150 \mathrm{pc}$. Several closer and much dispersed groups, such as TWA, have slightly different Galactic motion, but can be traced back to various parts of this conglomerate. It has a distinctly elongated shape in the sky plane projection (Sartori et al. 2003) stretching at a slight angle to the Galactic plane. The notion of propagating star formation along this chain of clouds proposed by de Geus et al. (1990) finds little evidence in the later studies devoted to this structure or its different parts. The median age of low-mass stars in US may be as low as $5 \mathrm{Myr}$ (Preibisch \& Zinnecker 1999), but we find Ophiuchus members as old as $14 \mathrm{Myr}$, still located within the boundaries of that 
association. The formation of the youngest stars outside the dense cloud is aligned with the eastern streamer, which looks more like a dynamic structure than a shock wave propagating from the direction of US (Fig. 5). The Scorpius-Centaurus OB associations are largely free of gas today, which has been interpreted as evidence for bygone supernova explosions. These hypothetical supernovae somehow failed to sweep up the gas in the nearby Ophiuchus region. If we assume that the star-forming gas in Ophiuchus, or some major part of it, was expelled from the Scorpio SFR by supernovae shock waves, a significant difference in space velocities should be present, and none has been found.

The kinematic pattern of star-forming regions in the fourth Galactic quadrant is enigmatic mostly because of its orderly character. We do not understand the origin of this order. Young stars inherit their Galactic orbits from the parent molecular cores, apart from postpartum dynamical interaction, which is negligible in sparse associations. Thus, the root of the problem is the orderly fashion in which the nearby molecular clouds move around the Sun. Taylor et al. (1987) investigated the conglomerate of nearby dark clouds and determined that the pattern of their radial velocities is consistent with the kinematics of the ringlike structure of OB stars known as the Gould Belt. Whether the Gould Belt is indeed a complete expanding ring of clouds or just a combination of extended chainlike complexes is not clear yet, but the highly organized motion of this conglomerate is obvious even in the allsky mapping of proper motion convergent points of X-ray active stars presented in (Makarov \& Urban 2000). A ringlike structure is unstable in the Galactic potential, but it is much easier to explain a long chain of gaseous clouds with a small internal velocity dispersion. It is well known that a system of bodies of limited initial velocity dispersion will remain confined in the radial $(X)$ and vertical $(Z)$ dimensions but will stretch out indefinitely in the tangential $(Y)$ dimension. Furthermore, this system will undergo periodic "focusing" events in the $X$ - $Z$ plane due to the epicyclic character of Galactic motion (Yuan 1977; Asiain et al. 1999;
Makarov et al. 2004). From our vantage point just outside the system, the clouds near the focusing point will be lined up in the $Y$-direction (possibly, with some inclination to the Galactic plane because of the initial systemic velocity in $Z$ ) and undergo a slight compression in the radial and vertical dimensions. Since the vertical and planar oscillations are slightly aperiodic, the focusing position may shift with time along the string of clouds (Yuan 1977). This simple dynamical phenomenon can account for the large-scale converging flows of clouds and protracted, spatially distributed star formation events. A concept of focusing, loosely collimated flow of molecular clouds finds support in the observed low velocity dispersions of local clouds and the apparent absence of energy equipartition over the mass range $10^{2}-10^{5.5} M_{\odot}$ (Stark 1984). Collision of two or more separate clouds at a moderate relative velocity $\left(\mathrm{a}\right.$ few $\mathrm{km} \mathrm{s}^{-1}$ ) can generate an agglomerate cloud with a high-density region suitable for star formation (Loren 1976). Stars formed in this region are expected to inherit a certain degree of kinematical segregation from the original clouds. The more accurate and deep astrometric catalogs and radial velocity surveys anticipated in the near future will be used to verify this model.

The research described in this paper was carried out at the Jet Propulsion Laboratory, California Institute of Technology, under a contract with the National Aeronautics and Space Administration. This research has made use of the SIMBAD database, operated at CDS, Strasbourg, France; and data products from the 2MASS, which is a joint project of the University of Massachusetts and the Infrared Processing and Analysis Center, California Technology Institute, funded by NASA and the NSF. I wish to thank Roc Cutri for pointing out the multicolor 2MASS calibration images available via the Web-based IRSA archive maintained at IPAC, and Solange Ramirez for help in finding and handling the data fro the $\mathrm{c} 2 \mathrm{~d}$ Spitzer Legacy program.
Adams, F., Lada, C., \& Shu, F. 1988, ApJ, 326, 865

Asiain, R., Figueras, F., \& Torra, J. 1999, A\&A, 350, 434

Ballesteros-Paredes, J., Hartmann, L., \& Vázquez-Semadeni, E. 1999, ApJ, 527, 285

Barrado y Navascués, D., et al. 2007, ApJ, 664, 481

Beichman, C., et al. 1986, ApJ, 307, 337

Bouvier, J., \& Appenzeller, I. 1992, A\&AS, 92, 481

Cieza, M., et al. 2005, ApJ, 635, 422

Colavita, M., et al. 2003, ApJ, 592, L83

de Bruijne, J. H. J. 1999, A\&A, 367, 111

de Bruijne, J. H. J., Hoogerwerf, R., \& de Zeeuw, P. T. 2001, A\&A, 367, 111

de Geus, E. J., Bronfman, L., \& Thaddeus, P. 1990, A\&A, 231, 137

de Zeeuw, P. T., Hoogerwerf, R., de Bruijne, J. H. J., Brown, A. G. A., \& Blaauw, A. 1999, AJ, 117, 354

Eggen, O. J. 1958, MNRAS, 118, 65

ESA. 1997, The Hipparcos and Tycho Catalogues (ESA SP-1200; ESA: Noordwijk)

Evans, N. J., et al. 2003, PASP, 115, 965

Famaey, B., et al. 2005, A\&A, 430, 165

Favata, F., et al. 1998, A\&A, 335, 218

Goodman, A. A., \& Arce, H. G. 2004, ApJ, 608, 831

Greene, T. P., \& Meyer, M. R. 1995, ApJ, 450, 233

Habart, E., et al. 2003, A\&A, 397, 623

Hartmann, L., Ballesteros-Paredes, J., \& Bergin, E. 2001, ApJ, 562, 852

Loren, R. B. 1976, ApJ, 209, 466

\section{REFERENCES}

Luhman, K. L., \& Rieke, G. H. 1999, ApJ, 528, 440

Makarov, V. V. 2006, AJ, 131, 2967

. 2007a, ApJ, 658, 480

2007b, ApJS, 169, 105

Makarov, V. V., Olling, R. P., \& Teuben, P. J. 2004, MNRAS, 352, 1199

Makarov, V. V., \& Robichon, N. 2001, A\&A, 368, 873

Makarov, V. V., \& Urban, S. 2000, MNRAS, 317, 289

Nordström, B., et al. 2004, A\&A, 418, 989

Mouschovias, T. C., et al. 2006, ApJ, 646, 1043

Palla, F., et al. 2005, ApJ, 626, L49

Preibisch, T., \& Zinnecker, H. 1999, AJ, 117, 2381

Ridge, N. A., et al. 2006, AJ, 131, 2921

Sartori, M. J., Lépine, J. R. D., \& Dias, W. S. 2003, A\&A, 404, 913

Siess, L., Dufour, E., \& Forestini, M. 2000, A\&A, 358, 593

Stark, A. A. 1984, ApJ, 281, 624

Strassmeier, K. G., et al. 2000, A\&AS, 142, 275

Tan, J. C. 2004, ApJ, 607, L47

Taylor, D. K., Dickman, R. L., \& Scoville, N. Z. 1987, ApJ, 315, 104

Weintraub, D. 1990, ApJS, 74, 575

Wilking, B. A., \& Lada, C. J. 1983, ApJ, 274, 698

Wilking, B. A., Schwartz, R. D., \& Blackwell, J. H. 1987, AJ, 94, 106

Wilking, B. A., et al. 2005, AJ, 130, 1733

Yuan, C. 1977, A\&A, 58, 53

Young, K. E., et al. 2006, ApJ, 644, 326

Zacharias, N., et al. 2004, AJ, 127, 3043 